\title{
Hsp27 silencing coordinately inhibits proliferation and promotes Fas-induced apoptosis by regulating the PEA-15 molecular switch
}

\author{
N Hayashi ${ }^{1}$, JW Peacock ${ }^{1}$, E Beraldi ${ }^{1}$, A Zoubeidi ${ }^{1,2}$, ME Gleave ${ }^{1,2}$ and CJ Ong ${ }^{\star, 1,3}$
}

Heat shock protein 27 (Hsp27) is emerging as a promising therapeutic target for treatment of various cancers. Although the role of Hsp27 in protection from stress-induced intrinsic cell death has been relatively well studied, its role in Fas (death domain containing member of the tumor necrosis factor receptor superfamily)-induced apoptosis and cell proliferation remains underappreciated. Here, we show that Hsp27 silencing induces dual coordinated effects, resulting in inhibition of cell proliferation and sensitization of cells to Fas-induced apoptosis through regulation of PEA-15 (15-kDa phospho-enriched protein in astrocytes). We demonstrate that Hsp27 silencing suppresses proliferation by causing PEA-15 to bind and sequester extracellular signal-regulated kinase (ERK), resulting in reduced translocation of ERK to the nucleus. Concurrently, Hsp27 silencing promotes Fas-induced apoptosis by inducing PEA-15 to release Fas-associating protein with a novel death domain (FADD), thus allowing FADD to participate in death receptor signaling. Conversely, Hsp27 overexpression promotes cell proliferation and suppresses Fas-induced apoptosis. Furthermore, we show that Hsp27 regulation of PEA-15 activity occurs in an Akt-dependent manner. Significantly, Hsp27 silencing in a panel of phosphatase and tensin homolog on chromosome 10 (PTEN) wild-type or null cell lines, and in LNCaP cells that inducibly express PTEN, resulted in selective growth inhibition of PTEN-deficient cancer cells. These data identify a dual coordinated role of Hsp27 in cell proliferation and Fas-induced apoptosis via Akt and PEA-15, and indicate that improved clinical responses to Hsp27-targeted therapy may be achieved by stratifying patient populations based on tumor PTEN expression.

Cell Death and Differentiation (2012) 19, 990-1002; doi:10.1038/cdd.2011.184; published online 16 December 2011

Heat shock protein 27 (Hsp27) is a 27-kDa molecular chaperone protein that is induced and phospho-activated in response to a variety of cytotoxic stressors, including hormonal, chemo- and radiotherapy. ${ }^{1}$ Increased expression of Hsp27 correlates with suppression of apoptosis and improved survival from a variety of cytotoxic insults. In cancer, Hsp27 is frequently overexpressed in numerous malignancies. $^{2,3}$ Elevated expression of Hsp27 in cancer has been associated with poor clinical prognosis and therapeutic resistance. Overexpression of $\mathrm{Hsp} 27$ in bladder, colon and prostate cancer cells enhances tumor growth and progression in vivo. ${ }^{4-6}$ Furthermore, selective inhibition of Hsp27 expression with antisense oligonucleotide (ASO)-based therapy has been shown to suppress tumor growth and sensitize cancer cells to hormonal, chemo- and radiotherapy. ${ }^{3-5,7}$ On the basis of these preclinical proof-of-principle studies, OGX-427, a selective, second-generation ASO inhibitor of Hsp27, has recently advanced into phase I/II clinical trials for treatment of a variety of cancers.
A better understanding of the precise molecular mechanisms by which Hsp27 controls cancer cell survival, death and proliferation may provide novel insights into the mechanism of action of Hsp27 antagonists in cancer therapy, and may facilitate the identification of patients who are most likely to benefit from treatment with Hsp27 inhibitors. Herein, we show that $\mathrm{Hsp} 27$ has a critical role in regulating cell proliferation and Fas (death domain containing member of the tumor necrosis factor receptor superfamily)-induced cell death. In the present study, we report that Hsp27 accelerates cell growth of prostate cancer cells by enhancing extracellular signalregulated kinase $(E R K)$ nuclear translocation via regulation of Akt activity and stability that, in turn, leads to increased phosphorylation and stabilization of PEA-15 (15-kDa phospho-enriched protein in astrocytes). Akt-mediated phosphorylation of PEA-15 alters the binding specificity of PEA-15, resulting in its dissociation from ERK alleviating cytoplasmic sequestration of ERK and promoting ERK nuclear translocation, ETS-like transcription factor 1 (Elk-1) activation and cell

\footnotetext{
${ }^{1}$ Vancouver Prostate Centre, University of British Columbia, Vancouver, British Columbia, Canada; ${ }^{2}$ Department of Urological Sciences, University of British Columbia, Vancouver, British Columbia, Canada and ${ }^{3}$ Department of Surgery, University of British Columbia, Vancouver, British Columbia, Canada

${ }^{*}$ Corresponding author: CJ Ong, Vancouver Prostate Centre, University of British Columbia, 2660 Oak Street, Vancouver, British Columbia V6H3Z6, Canada.

Tel: + 604875 4818; Fax: + 604875 5654; E-mail: chris.ong@ubc.ca

Keywords: prostate cancer; Hsp27; Akt; PEA-15/PED

Abbreviations: Ask1, apoptosis signal-regulating kinase 1; BrdU, bromodeoxyuridine; CHX, cycloheximide; Daxx, death-associated protein 6; CMV, cytomegalovirus; DAPI, 4',6'-diaminido-2-phenylindole; Dox, doxycycline; Elk-1, ETS-like transcription factor 1; ERK, extracellular signal-regulated kinase; FADD, Fas-associating protein with a novel death domain; Fas (also known as Apo-1 or CD95), death domain containing member of the tumor necrosis factor receptor superfamily; GFP, green fluorescent protein; GSK3, glycogen synthase kinase-3; Hsp27, heat shock protein 27; p27kip1, cyclin-dependent kinase inhibitor p27; PBS, phosphate-buffered saline; PEA-15, 15-kDa phospho-enriched protein in astrocytes; PI3K, phosphatidylinositol 3-kinase; PKB (also known as Akt), protein kinase B; PTEN, phosphatase and tensin homolog on chromosome 10; shRNA, short hairpin RNA; siRNA, small interfering RNA

Received 18.4.11; revised 17.10.11; accepted 10.11.11; Edited by P Vandenabeele; published online 16.12.11
} 
proliferation; meanwhile PEA-15 phosphorylation enhances its association with Fas-associating protein with a novel death domain (FADD) and suppresses Fas-induced apoptosis. Furthermore, we show that Hsp27 silencing selectively inhibits cell growth of phosphatase and tensin homolog on chromosome 10 (PTEN)-deficient cancer cells.

\section{Results}

Hsp27 accelerates LNCaP prostate cancer cell growth by promoting ERK nuclear translocation. The observation that Hsp27 overexpression leads to enhanced tumor growth and progression raises intriguing questions regarding whether Hsp27 might also be able to confer growthpromoting properties that contribute to the process of tumorigenesis, in addition to its cytoprotective functions. To further examine the effects of Hsp27 on cell growth, LNCaP cells were transduced with a lentiviral vector encoding an Hsp27 expression cassette $\left(\mathrm{LNCaP}_{\mathrm{Hsp27}}\right)$ or an empty vector $\left(\mathrm{LNCaP}_{\text {mock }}\right)$ as a control. As shown in Figure 1a, $\mathrm{LNCaP}_{\mathrm{Hsp} 27}$ cells expressed increased levels of Hsp27 protein that is primarily found in its phosphorylated, activated form. Consistent with previous findings, $\mathrm{LNCaP}_{\mathrm{Hsp} 27}$ cells exhibited enhanced cell growth as compared with $\mathrm{LNCaP}_{\text {mock }}$ (Figure 1a). To investigate whether Hsp27 improves cell growth, in part, by promoting cell cycle progression, cell proliferation of $\mathrm{LNCaP}_{\text {mock }}$ versus $\mathrm{LNCaP}_{\mathrm{Hsp} 27}$ cells was analyzed by assessing $\left[{ }^{3} \mathrm{H}\right]$-thymidine incorporation as a measure of DNA synthesis and by immunoblotting for panel of typical cell cycle markers. A greater extent of thymidine incorporation was observed in $\mathrm{LNCaP}_{\mathrm{Hsp} 27}$ than in $\mathrm{LNCaP}_{\text {mock }}$ cells, indicating that Hsp27 overexpression leads to an increased proportion of cells undergoing DNA synthesis (Figure 1b). Consistent with the enhanced DNA synthesis, $\mathrm{LNCaP}_{\mathrm{Hsp} 27}$ cells expressed increased levels of cyclin D1 and CDK2, with a concomitant decrease in cyclin-dependent kinase inhibitor p27 (p27kip1) expression (Figure 1c). Conversely, small interfering RNA (siRNA)-mediated silencing of Hsp27 inhibits LNCaP cell growth (Figure 1d). Collectively, these data support the notion that Hsp27 promotes cell proliferation.

Although large, ATP-dependent heat shock proteins, such as $\mathrm{Hsp} 90$ and Hsp70, have been shown to interact with many key regulatory components of the cell cycle machinery and the mitogenic signaling cascade, very little is known about the role of Hsp27 in cell proliferation. To assess whether Hsp27 regulates mitogenic signaling via the ERK/MAPK pathway, we assessed the activation status of the ERK/Elk-1 pathway in $\mathrm{LNCaP}_{\text {mock }}$ versus $\mathrm{LNCaP}_{\mathrm{Hsp} 27}$ cells. As shown in Figures 1e and $\mathrm{f}$, although $\mathrm{LNCaP}_{\mathrm{Hsp} 27}$ cells showed a slight/modest increase $(\sim 33 \%)$ above the already constitutively high levels of phospho-ERK seen in $\mathrm{LNCaP}_{\text {mock }}$ cells, a marked increase (two-fold) in Elk-1 activity was observed in $\mathrm{LNCaP}_{\mathrm{Hsp} 27}$ cells versus $\mathrm{LNCaP}_{\text {mock }}$ cells. The marked signal amplification that is observed suggests that Hsp27 may also function at an additional level downstream of ERK phosphorylation.

Upon phosphorylation, ERK translocates into the nucleus where it mediates its mitogenic effects. Hence, Hsp27 might potentially impinge on the ERK/MAPK pathway at the level of nuclear translocation of ERK. To examine whether Hsp27 regulates ERK translocation, the nucleocytoplasmic distribution of ERK was assessed in $\mathrm{LNCaP}_{\text {mock }}$ versus $\mathrm{LNCaP}_{\mathrm{Hsp} 27}$ cells by immunofluorescence microscopy. Indeed, $\mathrm{LNCaP}_{\mathrm{Hsp27}}$ cells exhibited increased localization of ERK in the nucleus (Figure $1 \mathrm{~g}$ ), suggesting that $\mathrm{Hsp} 27$ may regulate nuclear translocation of ERK.

Hsp27 regulates ERK translocation via PEA-15. A key regulator of ERK nuclear translocation is a small $15-\mathrm{kDa}$ cytoplasmic phosphoprotein, called PEA-15, that binds to and sequesters ERK in the cytoplasm in a phosphorylationregulated manner. ${ }^{8-10}$ Upon phosphorylation at Ser-116, PEA-15 dissociates from ERK, relieving the cytoplasmic sequestration of ERK and allowing its translocation to the nucleus. To test whether ERK translocation can be regulated by PEA-15 in a phosphorylation-dependent manner, LNCaP cells were transduced with a lentiviral vector expressing a non-phosphorylatable S116G point mutant of PEA-15, or a phosphomimetic S116D point mutant of PEA-15 or an empty vector as a control, and the nucleocytoplasmic distribution of ERK was visualized by immunofluorescence microscopy. Quantitative fluorescence imaging and immunoblotting analyses revealed that $\mathrm{LNCaP}$ cells expressing a nonphosphorylatable S116G point mutant of PEA-15 exhibited decreased levels of nuclear ERK compared with LNCaP cells expressing a phosphomimetic mutant of PEA-15 or empty vector-transduced control cells (Figures $2 a$ and b). Consistent with the levels of nuclear ERK, LNCaP cells expressing a non-phosphorylatable PEA-15 mutant displayed suppressed cell growth as compared with LNCaP cells expressing a phosphomimetic PEA-15 mutant or empty vector control cells (Figure 2c). Thus, these data support the notion that PEA-15 can regulate ERK translocation and cell proliferation in LNCaP cells in a phosphorylation-regulated manner.

Next, to assess whether ERK translocation in LNCaP cells is controlled in a PEA-15 regulated manner and whether Hsp27 regulates ERK translocation via PEA-15, the nucleocytoplasmic localization of ERK was analyzed in LNCaP cells stably transduced with a lentiviral vector encoding an short hairpin RNA (shRNA)-targeting PEA-15 (LN-221) or an empty lentiviral vector $\left(\mathrm{LNCaP}_{\text {mock }}\right)$ as control, and treated with either scrambled siRNA (Scr) or Hsp27 siRNA (siHsp27). Importantly, shRNA-mediated silencing of PEA-15 in LN-221 cells (Figure $2 \mathrm{~d}$ ) resulted in increased nuclear localization of ERK as compared with Scr-treated $\mathrm{LNCaP}_{\text {mock }}$ cells (Figure 2e), indicating that PEA-15 is a key regulator of ERK nuclear translocation in LNCaP cells. Furthermore, although siHsp27 treatment of $\mathrm{LNCaP}_{\text {mock }}$ cells resulted in reduced levels of ERK in the nucleus, the level of nuclear ERK remained high and unchanged upon siHsp27 treatment in PEA-15-knockdown LN-221 cells (Figures $2 d$ and e). Thus, these data indicate that $\mathrm{Hsp} 27$ regulates ERK translocation in a PEA-15-dependent manner.

Hsp27 interacts with and regulates the activity of Akt and PEA-15. Protein kinase B or PKB (also known as Akt) is a cell survival-promoting serine-threonine protein kinase that is activated in a phosphatidylinositol-3' kinase (PI3K)-dependent 
a

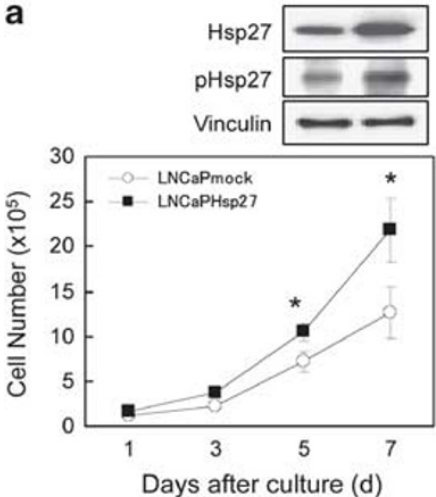

d

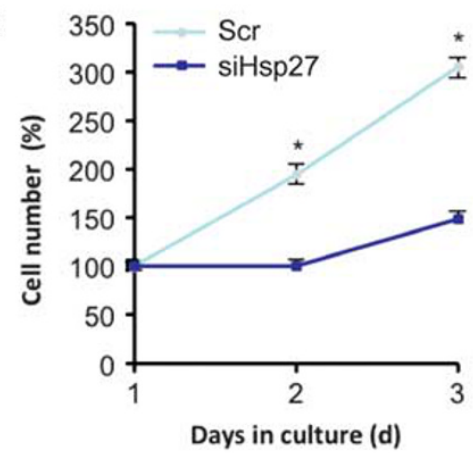

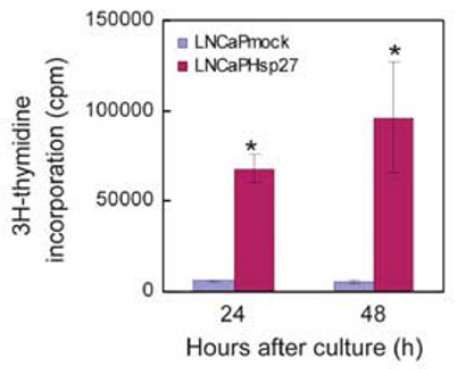

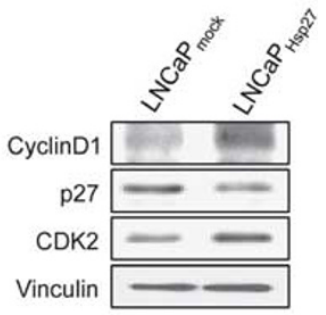

e
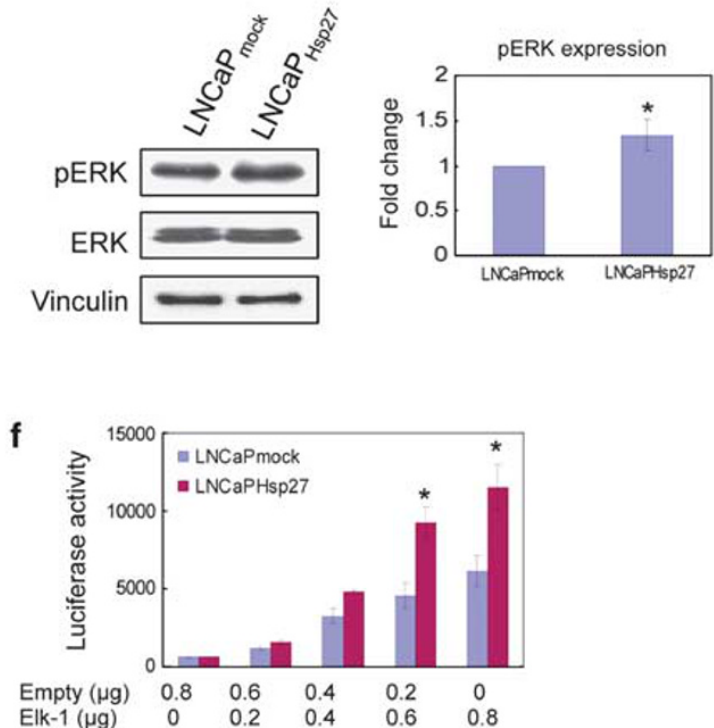

g

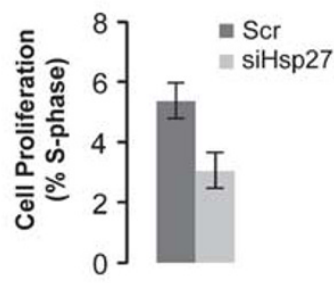

Elk-1 $(\mu \mathrm{g})$

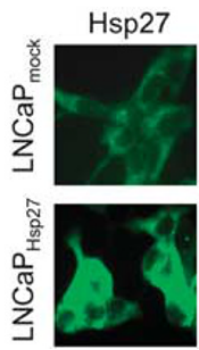

\section{ERK}

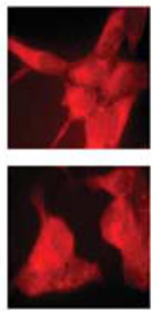

DAPI
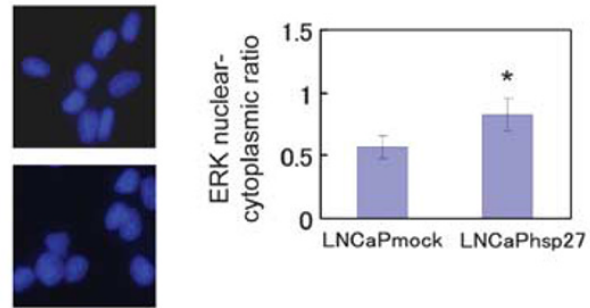

Figure 1 Hsp27 accelerates cell growth by promoting ERK nuclear translocation in LNCaP cells. (a) Hsp27 accelerates cell growth. Cell growth rates of LNCaP ${ }_{\text {Hsp27 }}$ and $\mathrm{LNCaP}_{\text {mock }}$ were compared by direct counting of viable cells. Levels of total and phospho-Hsp27 were analyzed by immunoblotting. (b) LNCaP $\mathrm{Hsp27}_{\mathrm{Cells}}$ exhibit enhanced proliferation. Cell proliferation of $\mathrm{LNCaP}_{\text {mock }}$ and $\mathrm{LNCaP}_{\mathrm{Hsp} 27}$ cells was assessed by $\left[^{3} \mathrm{H} \mathrm{H}\right.$-thymidine incorporation into DNA. The symbol (*) denotes statistical significance $(P<0.05)$. (c) $\mathrm{LNCaP}_{\mathrm{Hsp27}}$ cells display an expression profile of cell cycle markers that is consistent with increased cell proliferation. Cell lysates from $\mathrm{LNCaP}_{\text {mock }}$ and $\mathrm{LNCaP}_{\mathrm{Hsp27}}$ cells were analyzed for cyclin D1, p27kip1 and CDK2 by immunoblotting. Vinculin immunoblotting was performed as a loading control. (d) Hsp27 silencing inhibits LNCaP cell proliferation. Cell number was quantified using CyQuant proliferation assay (top), and percentage of cells undergoing DNA replication was determined by flow cytometric analyses of BrdU incorporation (bottom). (e) Overexpression of Hsp27 promotes ERK phosphorylation in LNCaP cells. Levels of phospho-ERK and total ERK protein were analyzed by immunoblotting. Vinculin immunoblotting was performed as a loading control. Relative levels of phospho-ERK expressions normalized to vinculin levels were analyzed in triplicate. The symbol ‘*' denotes statistical significance $(P<0.05)$. (f) $\mathrm{Hsp27}$ stimulates Elk-1 activity in $\mathrm{LNCaP}$ cells. LNCaP mock $_{\text {and }}$ aNCaP $_{\text {Hsp27 }}$ cells were transfected with the Elk-1 transcription reporter vector (pElk-1-GAL4) at various doses (0-0.8 $\mu \mathrm{g})$. At $48 \mathrm{~h}$ after transfection, the cell lysates were assayed for luciferase reporter activity. (g) Hsp27 overexpression enhances nuclear translocation of ERK. The nucleocytoplasmic distribution of ERK was assessed in LNCaP mock and LNCaP ${ }_{\text {Hsp27 }}$ cells by immunofluorescence microscopy. Cells were immunostained with anti-Hsp27 (green) and anti-ERK (red) antibodies. DAPI (blue) nuclear counterstaining was used to define the cell nuclei. The mean fluorescence ratios FN/C are shown for ERK. Values shown represent mean \pm S.D. The symbol ${ }^{(*)}$ denotes statistical significance $(P<0.05)$. The results $(\mathbf{a}, \mathbf{b}, \mathbf{d}$ and $\mathbf{e})$ are the means of three independent experiments 
a
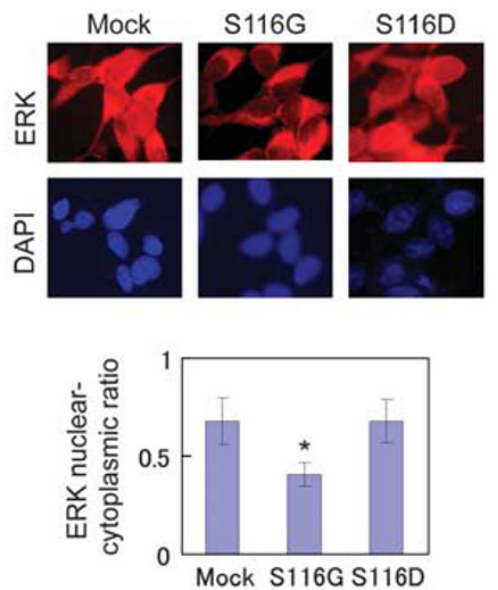

C

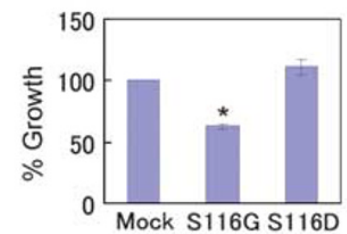

e

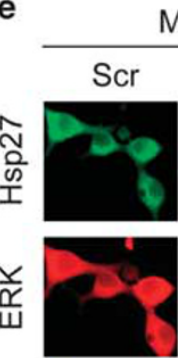

Mock

iHsp27

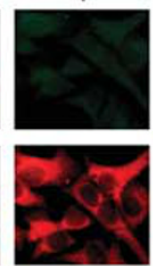

高
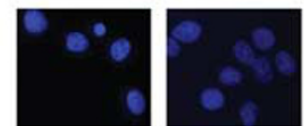

LN-221

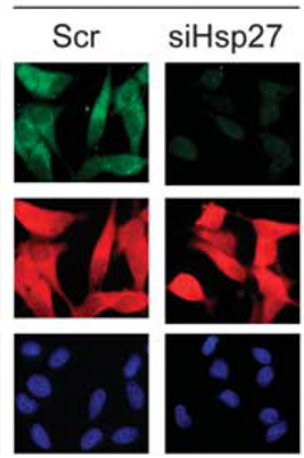

b
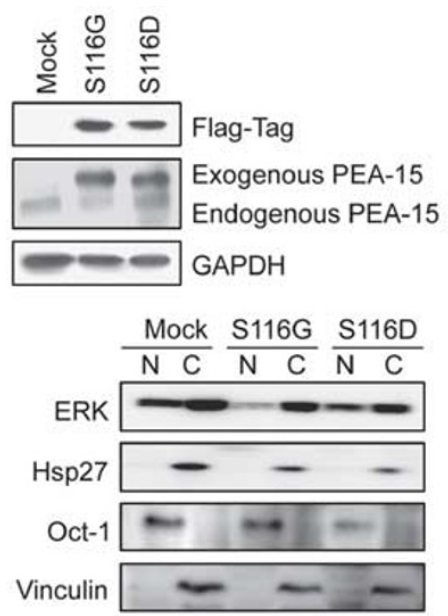

d

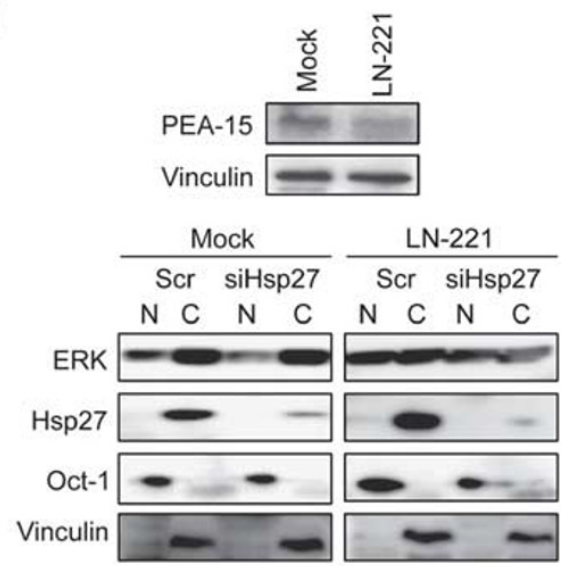

Figure 2 ERK translocation is regulated by PEA-15 in phosphorylation-dependent manner in LNCaP cells. (a) PEA-15 regulates ERK translocation. LNCaP cells transfected with a non-phosphorylatable mutant (S116G) or a phosphomimetic mutant of PEA-15 (S116D). The nucleocytoplasmic distribution of ERK was assessed in LNCaP mock $_{\text {and }}$ $\mathrm{LNCaP}_{\mathrm{Hsp27}}$ cells by immunofluorescence microscopy. Cells were immunostained with anti-ERK (red) antibodies. DAPI (blue) nuclear counterstaining was used to define the cell nuclei. (b) Levels of exogenous and endogenous PEA-15 proteins were analyzed by immunoblotting with anti-Flag and anti-PEA-15 antibodies. (c) Growth effect of LNCaP cells expressing non-phosphorylatable (S116G) and phosphomimetic (S116D) PEA-15 mutants. Cell growth rates of LNCaP cells were compared by crystal violet assay. (d) Protein levels of ERK, Hsp27, Oct-4 and vinculin were determined in nuclear and cytoplasmic fractions of cell lysates derived from LN-221 versus LNCaP mock cells treated with Scr or siHsp27 by immunoblotting. shRNA-mediated silencing of PEA-15 was examined by immunoblotting. (e) ERK nuclear translocation in LNCaP cells is regulated by PEA-15. The nucleocytoplasmic localization of ERK was analyzed in $\mathrm{LN}-221$ versus $\mathrm{LNCaP}_{\text {mock }}$ cells treated with Scr or siHsp27 by immunofluorescence microscopy as described above. Cells were immunostained with anti-Hsp27 (green) and anti-ERK (red) antibodies. DAPI (blue) nuclear counterstaining was used to define the cell nuclei. The mean fluorescence ratios FN/C are shown for ERK. Values shown represent mean \pm S.D. The symbol ${ }^{(* \prime}$ denotes statistical significance $(P<0.05)$

manner. ${ }^{11}$ Hsp27 has previously been shown to bind and activate $\mathrm{PKB} /$ Akt in neutrophils. Furthermore, Beguinot and coworkers have shown that $\mathrm{PKB} / \mathrm{Akt}$ binds, stabilizes and phosphorylates PEA-15 at Ser-116, regulating its activity. ${ }^{12}$ Therefore, we explored the possibility that Hsp27 may indirectly regulate PEA-15 by stabilizing and activating Akt.
To examine whether changes in Hsp27 expression levels could modulate total Akt and PEA-15 expression levels as well as Akt activation and PEA-15 phosphorylation, the levels of PEA-15, phospho-PEA-15, Akt, phospho-Akt Ser-473 and phospho-Foxo-1 were analyzed by immunoblotting in $\mathrm{LNCaP}_{\text {mock }}$ versus $\mathrm{LNCaP}_{\mathrm{Hsp} 27}$ cells (Figure $3 \mathrm{a}$ ). In addition, 
a

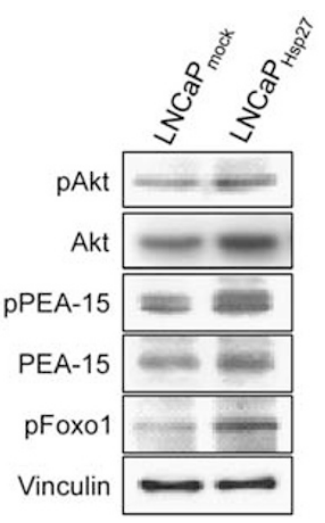

C

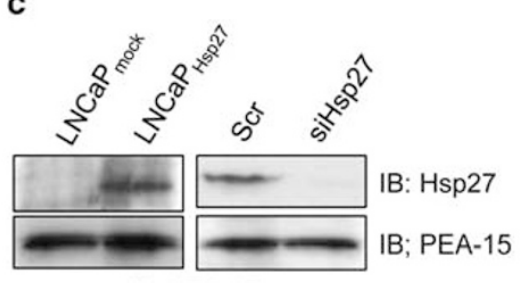

IP: PEA-15

b

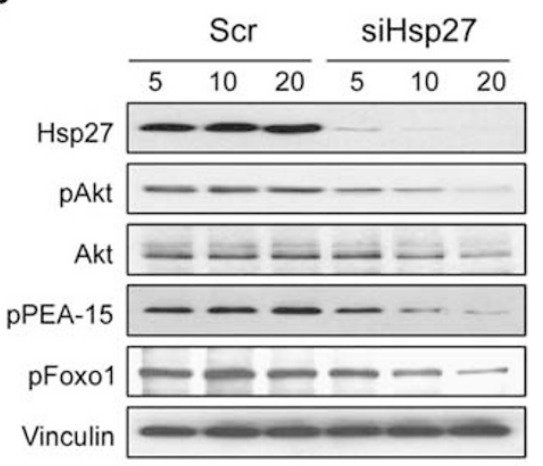

d

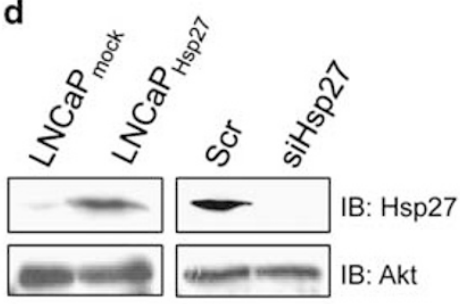

IP: Akt

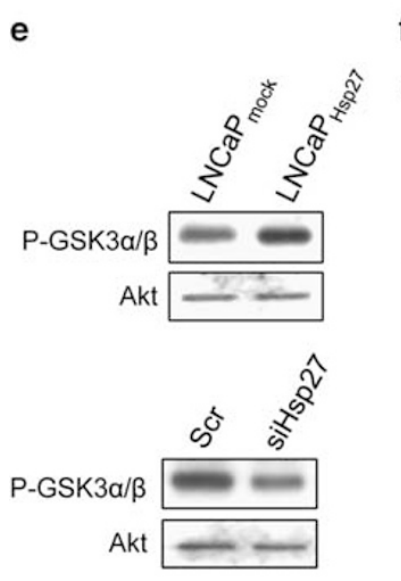

f

Scr

siHsp27

$\mathrm{LNCaP}_{\text {mock }} \mathrm{LNCaP}_{\mathrm{Hsp} 27}$

$\begin{array}{lllllllllllllllllll}\mathrm{CHX}(\mathrm{h}) & 0 & 2 & 8 & 18 & 0 & 2 & 8 & 18 & & 0 & 2 & 8 & 18 & 0 & 2 & 8 & 18\end{array}$
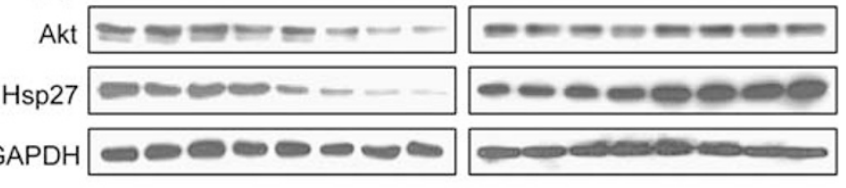

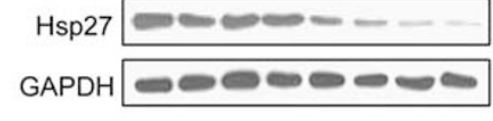
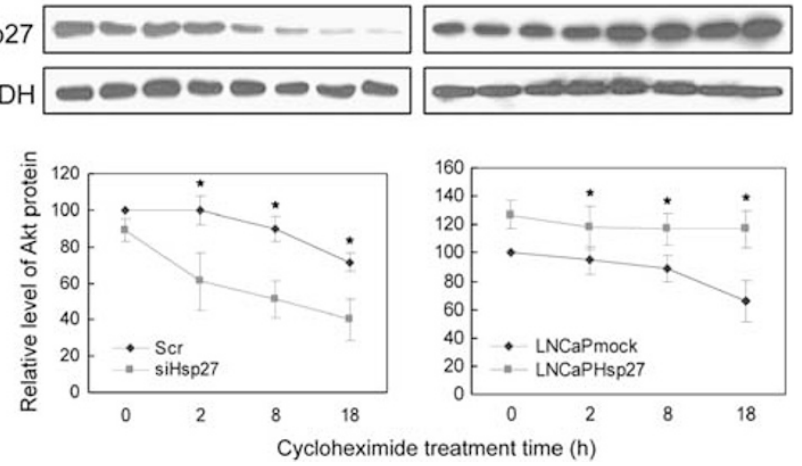

Figure 3 Hsp27 regulates PEA-15 phosphorylation in an Akt-dependent manner in LNCaP cells. (a) Constitutive overexpression of Hsp27 induces increased phosphorylation of PEA-15 at Ser-116. Cell lysates derived from $\mathrm{LNCaP}_{\text {mock }}$ and $\mathrm{LNCaP}_{\mathrm{Hsp27}}$ cells were analyzed for total Akt, phospho-Akt (Ser-473), total PEA-15, phosphoPEA-15 (Ser-116) and phospho-Foxo-1 (Ser-256) by immunoblotting. Vinculin immunoblotting was performed as a loading control. (b) siRNA-mediated silencing of Hsp27 leads to decreased levels of phospho-PEA-15 (Ser-116). LNCaP cells were transfected with various doses (5-20 nM) of Scr or siHsp27. Forty-eight hours after transfection, immunoblotting was performed to analyze the total amounts and phosphorylation states of the indicated proteins. (c and d) Hsp27 interacts with Akt and PEA-15. Lysates from $\mathrm{LNCaP}_{\text {mock }}$ or $\mathrm{LNCaP}_{\mathrm{Hsp} 27}$ cells or from LNCaP cells following transfection with $20 \mathrm{nM}$ Scr or siHsp27 for $48 \mathrm{~h}$ were subjected to anti-PEA-15 or anti-Akt immunoprecipitation (IP) and subsequently immunoblotted (IB) with either anti-Hsp27 and anti-PEA-15, or anti-Hsp27 and anti-Akt antibodies. (e) Hsp27 regulates Akt activity. In vitro Akt kinase assays were performed using the Akt kinase assay kit (Cell Signaling Technology). (f) Hsp27 regulates Akt protein stability. Cells were treated with $10 \mu \mathrm{g} / \mathrm{ml} \mathrm{CHX}$ for the indicated times. DMSO was used as a control. Akt and Hsp27 protein levels were analyzed by immunoblotting. Graphs represent densitometric analyses of the levels of total Akt protein from cells treated with $\mathrm{CHX}$ for varying lengths of time. Data are presented as means \pm S.D. from three independent experiments (lower panel). The symbol '*' denotes statistical significance $(P<0.05)$

the levels of the indicated proteins were examined in LNCaP cells treated with Scr versus siHsp27 (Figure 3b).

As shown in Figure 1a, $\mathrm{LNCaP}_{\mathrm{Hsp} 27}$ cells exhibited elevated levels of Hsp27 and phospho-Hsp27 (Ser-82), a marker of Hsp27 activation status. The overexpression of Hsp27 in $\mathrm{LNCaP}_{\mathrm{Hsp27}}$ cells was associated with increased levels of total Akt and phospho-Akt (Ser-473) (a marker of Akt activation status), total PEA-15, phospho-PEA-15 (Ser-116) and phospho-Foxo-1 (a bona fide substrate of Akt as a marker of Akt activity ${ }^{11}$; Figure 3a). Conversely, Hsp27 silencing led to decreased levels of the indicated proteins and phosphoproteins in a dose-dependent manner (Figure 3b). These data are consistent with the notion that $\mathrm{Hsp} 27$ may regulate PEA-15 by activating Akt, which, in turn, leads to stabilization and phosphorylation PEA-15.

Next, we examined whether Hsp27 and PEA-15 exist in the same protein complex. PEA-15 was immunoprecipitated from $\mathrm{LNCaP}_{\text {mock }}$ versus $\mathrm{LNCaP}_{\mathrm{Hsp27}}$ cells, and from 
siHsp27- versus Scr- treated LNCaP cells, and the presence of Hsp27 analyzed by immunoblotting. As shown in Figure 3c, increased amounts of Hsp27 coprecipitated with PEA-15 from Hsp27-overexpressing cells, and conversely less Hsp27 was detected in immunoprecipitates from Hsp27-silenced cells (Figure $3 \mathrm{c}$ ). These results indicate that Hsp27 interacts, either directly or indirectly, with PEA-15.

To determine whether Hsp27 physically interacts with Akt in LNCaP cells, similar co-immunoprecipitation (co-IP) experiments were performed. Overexpression of Hsp27 increased the amount of Hsp27 that co-immunoprecipitates with Akt, whereas Hsp27 knockdown diminished this interaction (Figure 3d). To examine whether this interaction is associated with changes in Akt activity, in vitro Akt kinase assays were performed. Similar amounts of Akt were immunoprecipitated from each cell type and subjected to Akt kinase activity assessment using glycogen synthase kinase-3 $\alpha / \beta(\mathrm{GSK} 3 \alpha / \beta)$ as a substrate. As shown in Figure 3e, Hsp27 silencing decreased Akt activity, whereas increased Akt activity was observed in Hsp27-overexpressing $\mathrm{LNCaP}_{\mathrm{Hsp} 27}$ cells.

The effect of Hsp27 expression on Akt protein half-life was then evaluated. Cycloheximide $(\mathrm{CHX})$ was added to $\mathrm{LNCaP}_{\text {mock}}$, $\mathrm{LNCaP}_{\mathrm{Hsp} 27}$ and LNCaP cells transfected with Scr or siHsp27 to block de novo protein synthesis, and the levels of Akt protein were monitored by immunoblotting. As shown in Figure 3f, Akt protein levels rapidly decreased after $\mathrm{CHX}$ treatment in Hsp27-knockdown cells. In contrast, Hsp27 overexpression prolonged Akt half-life compared with LNCaP cells with empty vector transfected controls. Taken together, these data suggest that Hsp27 directly interacts with and stabilizes Akt, and regulates its activity.

To determine whether Akt is the primary kinase responsible for regulating the phosphorylation of PEA-15 on Ser-116 in LNCaP cells, the effect of Akt inhibition using an Akt-specific siRNA, Akt siRNA (siAkt) or a potent small-molecule Akt inhibitor, GSK690693C, ${ }^{13}$ on PEA-15 Ser-116 phosphorylation were assessed by western blot analyses. siAkt treatment downregulated the levels of Akt, phospho-Akt, PEA-15, and phospho-PEA-15, whereas GSK690693C treatment had a dose-dependent decrease of phospho-GSK3 $\beta$ and phosphoPEA-15, accompanied by upregulation of phospho-Akt (Figures $4 a$ and b). Consistent with previous reports, ${ }^{12}$ these data indicate that Akt can regulate PEA-15 phosphorylation and stability, and furthermore, suggest that Akt is the principle kinase that is responsible for phosphorylating PEA-15 at Ser116 in LNCaP cells.

To test whether Akt activity can regulate ERK translocation, LNCaP cells were treated with or without GSK690693C, and the nucleocytoplasmic localization of ERK was assessed by immunofluorescence microscopy. As shown in Figure 4c, treatment of LNCaP cells with GSK690693C suppressed nuclear translocation of ERK, suggesting that Akt can regulate ERK nuclear translocation.

Hsp27 regulates induction of Fas-induced apoptosis in LNCaP cells. PEA-15 has been identified as a key molecular switch that regulates cell growth and cell death. PEA-15 switches its binding affinities for either FADD or ERK a

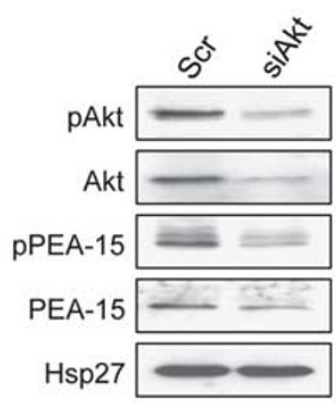

C

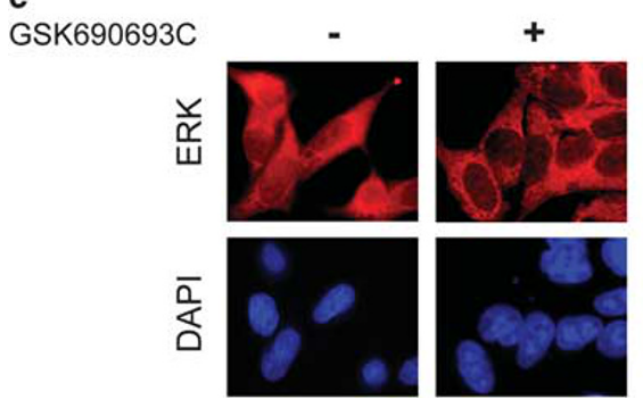

b
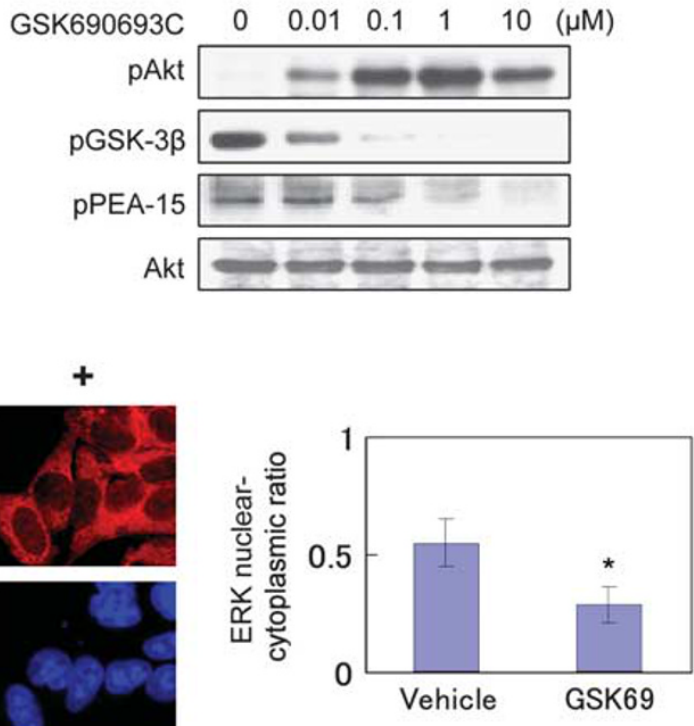

Figure 4 Phosphorylation of PEA-15 at Ser-116 occurs in an Akt-dependent manner in LNCaP cells. (a) Akt silencing downregulates phospho-PEA-15 expression. $\mathrm{LNCaP}$ cells were treated with $100 \mathrm{nM} \mathrm{Scr}$ or siAkt for $24 \mathrm{~h}$. Forty-eight hours after transfection, protein lysates were analyzed by immunoblotting with the indicated antibodies. (b) LNCaP cells were treated with various concentrations of the potent small-molecule Akt inhibitor, GSK690693C, or control (DMSO). Twenty-four hours after treatment, total amounts and phosphorylation states of the indicated proteins were analyzed by immunoblotting. (c) GSK690693C inhibits the nuclear localization of ERK. Nuclear translocation of ERK was assessed by immunofluorescence microscopy $24 \mathrm{~h}$ after treatment with $1 \mu \mathrm{M}$ GSK690693C or control (DMSO). Cells were immunostained with antiERK (red) antibodies. DAPI (blue) nuclear counterstaining was used to define the cell nuclei. The mean fluorescence ratios FN/C are shown for ERK. Values shown represent mean \pm S.D. The symbol ‘*' denotes statistical significance $(P<0.05)$ 
depending on its phosphorylation status. ${ }^{14}$ Upon phosphorylation of PEA-15 at Ser-116, PEA-15 releases ERK and binds to FADD. Phospho-PEA-15 mediates its antiapoptotic effects by directly binding to and inhibiting FADD. Thus, the ability of Hsp27 to regulate PEA-15 phosphorylation predicts that $\mathrm{Hsp} 27$ may also modulate Fas-induced apoptosis via PEA-15/FADD. Therefore, we examined the effects of Hsp27 on Fas-induced apoptosis in LNCaP cells.

LNCaP cells are intrinsically insensitive to Fas-induced apoptosis, but can be sensitized by treatment with $\mathrm{CHX} .^{15}$
To determine whether Hsp27 can regulate Fas-induced apoptosis, $\mathrm{LNCaP}_{\text {mock }}$ and $\mathrm{LNCaP}_{\mathrm{Hsp} 27}$ cells were treated with $1.0 \mu \mathrm{g} / \mathrm{ml}$ anti-Fas antibody, $\mathrm{CH}-11$, in the presence or absence $2.5 \mu \mathrm{g} / \mathrm{ml} \mathrm{CHX}$. CH-11 treatment had no effect on $\mathrm{LNCaP}_{\text {mock }}$ and $\mathrm{LNCaP}_{\mathrm{Hsp} 27}$ cells on its own, but induced apoptosis when it was combined with $\mathrm{CHX}$ (Figure 5a). However, Hsp27 overexpression in $\mathrm{LNCaP}_{\mathrm{Hsp} 27}$ cells markedly suppressed $\mathrm{CH}-11$-induced apoptosis. Conversely, Hsp27 knockdown by siHsp27 sensitized LNCaP cells to $\mathrm{CH}$-11-induced apoptosis in the absence of $\mathrm{CHX}$ (Figure 5b). To determine whether the ability of Hsp27 to suppress
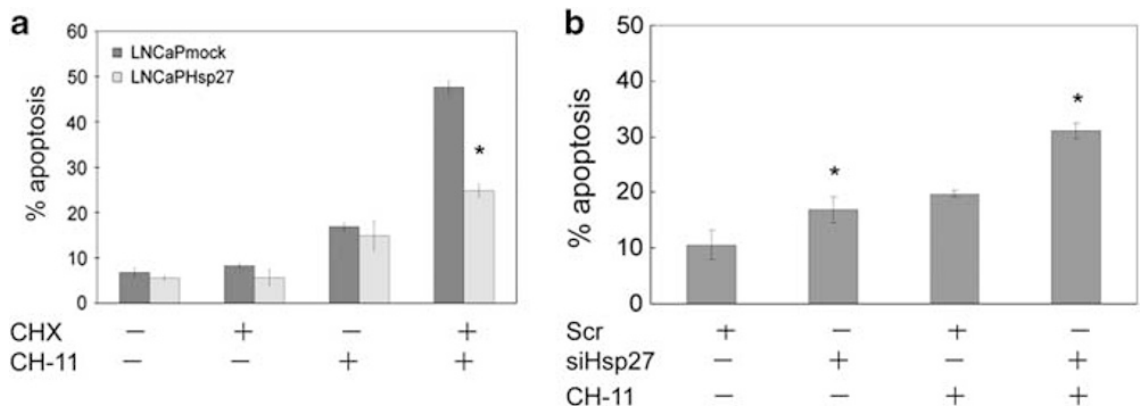

C

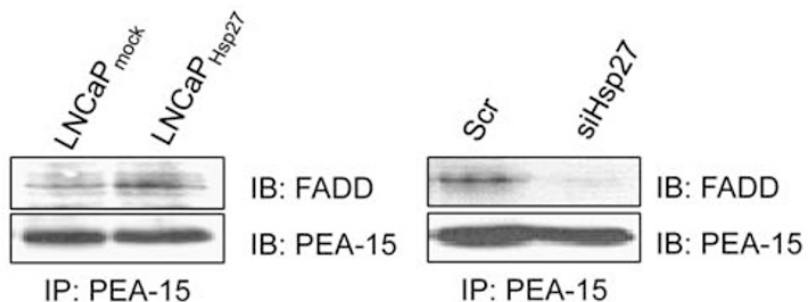

d

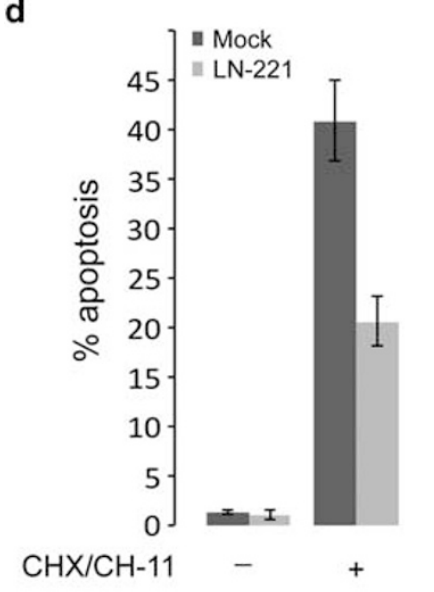

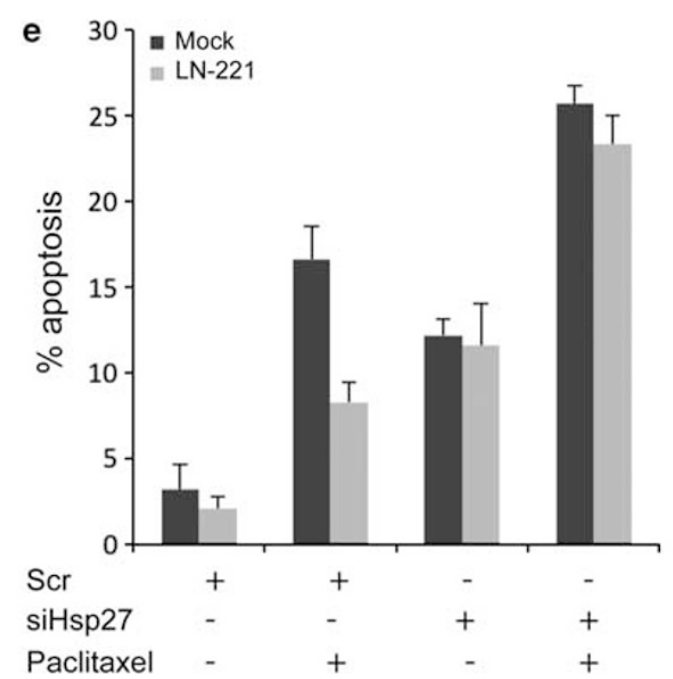

Figure 5 Hsp27 regulates Fas-induced apoptosis by modulating FADD binding to PEA-15. (a) Hsp27 suppresses Fas-induced apoptosis. LNCaP mock $_{\text {and LNCaP }}$ Hsp27 cells were treated with either $2.5 \mu \mathrm{g} / \mathrm{ml} \mathrm{CHX}$ alone, $1.0 \mu \mathrm{g} / \mathrm{ml}$ agonistic anti-Fas antibody (CH-11) alone or in combination in $5 \%$ charcoal-stripped serum media. Apoptosis was assessed by measuring the percentage cells containing subdiploid DNA (\% sub-G1) as determined by propidium iodide staining and flow cytometry $24 \mathrm{~h}$ after treatment. (b) Hsp27 silencing promotes Fas-induced apoptosis. At $48 \mathrm{~h}$ after transfection of LNCaP cells with $20 \mathrm{nM} \mathrm{Scr}$ or $20 \mathrm{nM}$ siHsp27, apoptosis in response to $\mathrm{CH}-11$ was

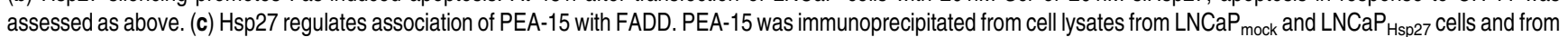
LNCaP cells transfected with $20 \mathrm{nM}$ Scr or $20 \mathrm{nM}$ siHsp27 and cultured for $24 \mathrm{~h}$. Immune complexes were resolved on SDS-PAGE and immunoblotted for FADD and PEA-15.

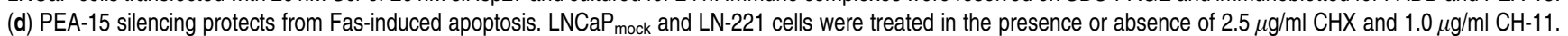
Apoptosis was assessed $24 \mathrm{~h}$ after treatment. (e) Role of PEA-15 in intrinsic apoptosis. LNCaP mock $_{\text {and }} \mathrm{LN}-221$ cells were treated with Scr or siHsp27 in the presence or absence of paclitaxel. Percentage of cells undergoing apoptosis was measured as above. The results $(\mathbf{a}, \mathbf{b}, \mathbf{d}$ and $\mathbf{e})$ are the means of three independent experiments. Values shown represent mean \pm S.D. The symbol “*' denotes statistical significance $(P<0.05)$ 
Fas-induced apoptosis is associated with the interaction of PEA-15 with FADD, PEA-15 co-IP assays were performed. Hsp27 knockdown diminished the interaction of PEA-15 with FADD, whereas increased Hsp27 enhanced this association (Figure 5c). Furthermore, PEA-15 silencing suppressed Fasinduced apoptosis of LNCaP cells (Figure $5 \mathrm{~d}$ ). To determine the role of PEA-15 and Hsp27 in regulating apoptosis by paclitaxel - an inducer of intrinsic apoptosis - $\mathrm{LNCaP}_{\text {mock }}$ versus PEA-15-silenced LN-221 cells were treated with Scr or siHsp27 \pm paclitaxel (Figure 5e). As expected, PEA-15 silencing protected from paclitaxel-induced apoptosis in Scrtreated cells, as PEA-15 silencing leads to constitutive ERK activation that has been shown to promote prosurvival signals through multiple mechanisms, such as phosphorylation of $B A D$ and induction of CREB-mediated transcription of prosurvival genes. ${ }^{16}$ Furthermore, apoptosis induced by Hsp27 silencing occurs in a PEA-15-independent manner, and PEA-15 silencing does not block Hsp27's cytoprotective activity against apoptosis induced through an intrinsic apoptotic pathway (Figure 5e). These data collectively suggest that Hsp27 may regulate Fas-induced apoptosis by modifying the interaction of PEA-15 with FADD in LNCaP cells.

Effects of Hsp27 knockdown in PC-3 cells. To determine whether the effects of Hsp27 on Akt and PEA-15 are not unique to LNCaP cells, we tested whether these Hsp27mediated changes would hold true on a second
PTEN-deficient prostate cancer cell line, PC-3 cells. As with LNCaP cells, siHsp27-mediated silencing resulted in decreased expression levels of total Akt, phospho-Akt (Ser473), phospho-PEA-15 (Ser-116) and phospho-Foxo-1 (Ser256) in dose-dependent manner in PC-3 cells (Figure 6a). In addition, as observed in LNCaP cells, ERK nuclear accumulation clearly decreased after siHsp27 treatment, correlating with decreased phospho-PEA-15 expression level (Figure 6b). Finally, siHsp27 treatment sensitized Fasresistant PC-3 cells to Fas-induced apoptosis in PC-3 cells as observed previously in LNCaP cells (Figure 6c).

Hsp27 silencing selectively inhibits PTEN-deficient cancer cells. On the basis of our findings described above that Hsp27 regulates cell proliferation through PEA-15 in an Akt-regulated manner, we hypothesized that the growth inhibitory effects of $\mathrm{Hsp} 27$ silencing may be more pronounced in cells that have an activated PI3K/Akt pathway. The PTEN tumor suppressor gene is among the most commonly mutated genes in cancer. ${ }^{17}$ PTEN is a lipid phosphatase, which is a key negative regulator of the PI3K/ Akt cell growth and survival pathway. Loss of PTEN confers constitutive activation of the PI3K/Akt pathway, and many cancer cells harboring PTEN mutations become exquisitely dependent on the PI3K/Akt pathway for their growth and survival - a phenomena referred to as 'pathway or oncogene addiction'. ${ }^{18,19}$ To determine whether PTEN expression status is an important predictor for growth inhibition by

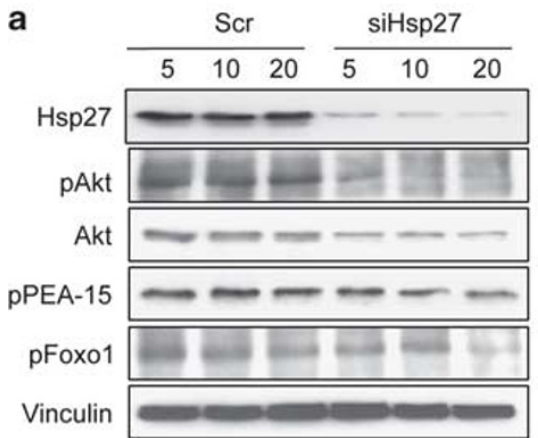

b
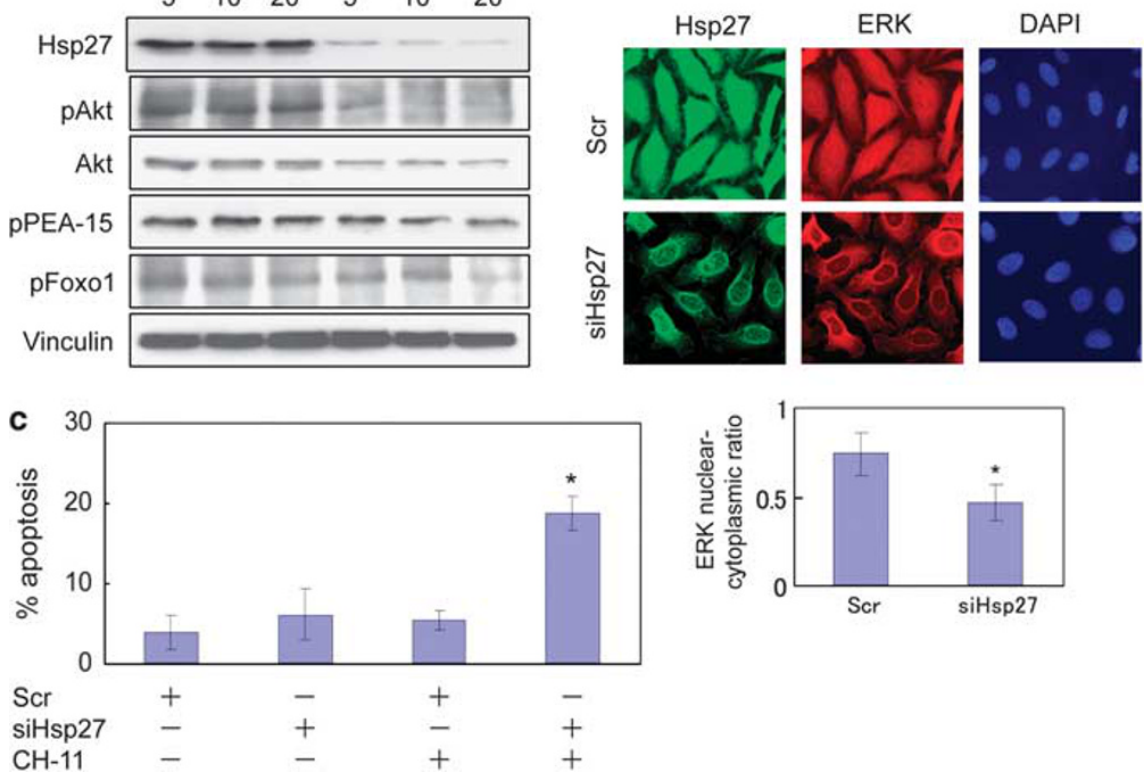

Figure 6 Hsp27 regulates phosphorylation status of Akt and PEA-15, nuclear translocation of ERK and Fas-induced apoptosis in androgen-independent PC-3 cells. (a) PC-3 cells were transfected with various doses $(5-20 \mathrm{nM})$ of Scr or siHsp27. Forty-eight hours after transfection, immunoblotting was performed to analyze the total amounts and phosphorylation states of the indicated proteins. (b) Nuclear translocation of ERK was assessed by immunofluorescence microscopy after transfection of PC-3 cells with $20 \mathrm{nM} \mathrm{Scr}$ or $20 \mathrm{nM}$ siHsp27. Cells were fixed $18 \mathrm{~h}$ after culturing in low-serum $(0.5 \%)$ conditions and were assessed by immunofluorescence staining with antiHsp27 (green) and anti-ERK (red) antibodies. DAPI (blue) nuclear counterstaining was used for marking cell nuclei. The mean fluorescence ratios FN/C are shown for ERK. Values shown represent mean \pm S.D. The symbol *' denotes statistical significance $(P<0.05)$. (c) Assessment of the effect of Hsp27 knockdown on Fas-induced apoptosis of PC-3 cells. PC-3 cells were transfected with $20 \mathrm{nM}$ Scr or $20 \mathrm{nM}$ siHsp27. At $48 \mathrm{~h}$ after transfection, apoptosis in response to the agonistic anti-Fas antibody, $\mathrm{CH}-11$, was assessed by flow cytometric analyses of the proportion of cells containing subdiploid DNA as described above 
Hsp27 silencing, the effect of siHsp27 treatment on the growth rates of seven PTEN-positive and five PTEN-deficient cancer cell lines was examined. siHsp27 treatment significantly inhibited cell growth of PTEN-deficient cancer cells, whereas growth rates of PTEN-positive cells were relatively unaffected by $\mathrm{Hsp} 27$ silencing (Figure 7a). Interestingly, PTEN-negative cells also tended to exhibit increased levels of Hsp27 as compared with PTEN-positive cells (Figure 7a).
As shown in Figure 7b, siHsp27 treatment led to significant downregulation of $\mathrm{Hsp} 27$ expression in PTEN-positive BPH-1 and PTEN-negative MDA468 cells. As expected, PTENdeficient MDA468 cells expressed high levels of phospho-Akt, whereas PTEN-positive BPH-1 cells expressed low basal phospho-Akt levels. Furthermore, siHsp27 treatment resulted in significant downregulation of phospho-Akt and phosphoPEA-15 in MDA468 cells, whereas phospho-Akt and phospho-PEA-15 levels remained low in $\mathrm{BPH}-1$ cells (Figure $7 \mathrm{~b}$ ).
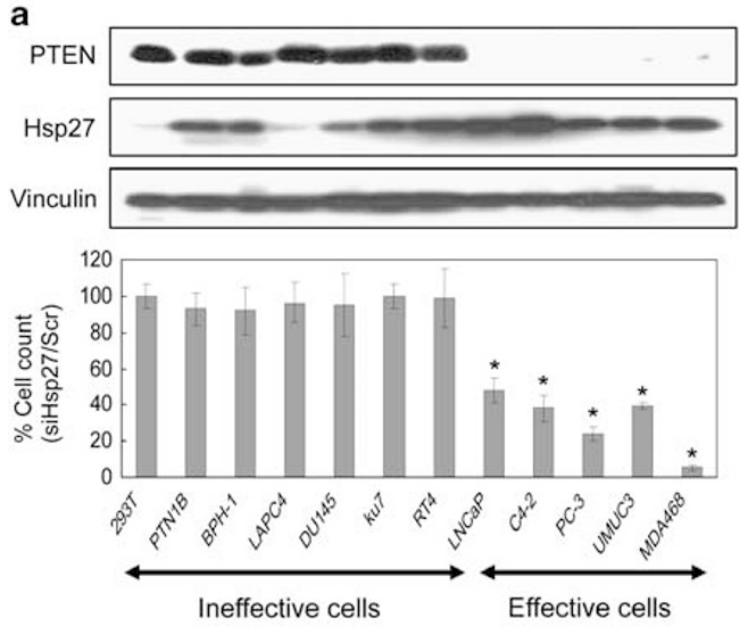

C

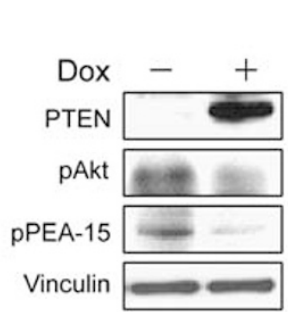

d

\section{d}

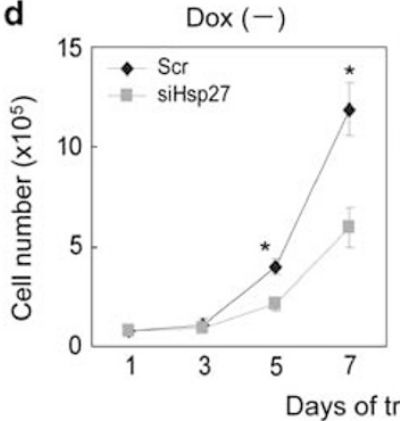

b

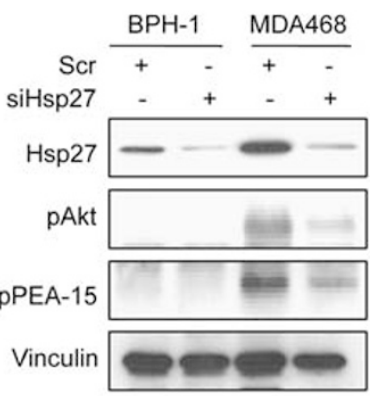

e

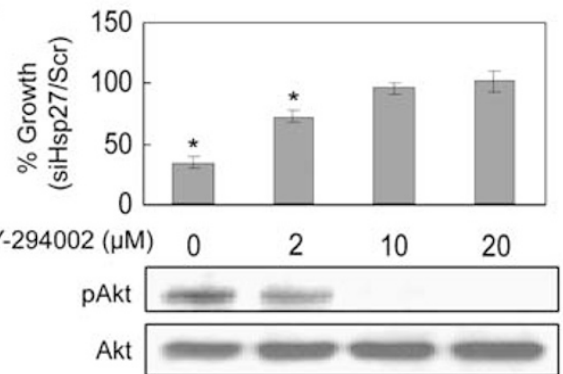

Figure 7 Hsp27 knockdown selectively inhibits growth of PTEN-deficient cells. (a) The effects of Hsp27 knockdown on cell growth were compared among 12 cell lines after transfection with $20 \mathrm{nM}$ Scr or $20 \mathrm{nM}$ siHsp27. The determination of cell proliferation was achieved by direct cell count at 2-day intervals up to day 7 after transfection. The date of transfection was considered as day 1. Data are presented as percentage of control. Cell numbers of control cells treated with Scr were considered as $100 \%$. The levels of expression of PTEN and Hsp27 in each of the cell lines were determined by immunoblotting using the indicated antibodies as described above. Vinculin immunoblotting was performed as a protein loading control. (b) The levels of Hsp27, phospho-Akt and phospho-PEA-15 in BPH-1 and MDA468 transfected with $20 \mathrm{nM}$ Scr or siHsp27 were assessed by immunoblotting. (c) Cell lysates from PTEN Tet-on LNCaP cells treated in the presence or absence of $1 \mu \mathrm{g} / \mathrm{ml}$ Dox for $24 \mathrm{~h}$ were analyzed for levels of PTEN, phospho-Akt and phospho-PEA-15 by immunoblotting. (d) Twenty-four hours after preculturing with or without of Dox, cells were transfected with $20 \mathrm{nM}$ Scr or siHsp27, and cell growth was determined at the indicated times by direct viable cell counting. (e) Growth inhibitory effect mediated by Hsp27 knockdown in PC-3 cells after PI3K blockade with LY-294002 was determined by staining cell protein with crystal violet and measuring absorbance at $595 \mathrm{~nm}$. After transfection with $20 \mathrm{nM}$ Scr or siHsp27, PC-3 cells were treated with various doses of LY-294002 $(2-20 \mu \mathrm{M})$ or control (DMSO). Absorbance of control treated with Scr was considered as $100 \%$. The results (a, $\mathbf{d}$ and $\mathbf{e})$ are the means of three independent experiments. Values shown represent mean \pm S.D. The symbol "*' denotes statistical significance $(P<0.05)$ 
To determine whether the ability of Hsp27 silencing to inhibit cell growth is dependent on PTEN expression, we used the LNCaP PTEN Tet-on cells that express PTEN in a doxycycline (Dox)-inducible manner. Dox treatment of LNCaP PTEN Teton cells resulted in the induction of PTEN expression that was associated with a concomitant downregulation of phospho-Akt and phospho-PEA-15 levels (Figure 7c). In the absence of Dox, siHsp27 treatment of PTEN-deficient LNCaP PTEN Tet-on cells resulted in significant inhibition of cell growth, whereas upon Dox treatment the growth inhibitory effect induced by Hsp27 silencing was abrogated in PTEN-expressing LNCaP PTEN Tet-on cells (Figure 7d). In a parallel set of experiments, to test whether the activity of PI3K influences the magnitude of the growth inhibitory effects induced by Hsp27 silencing, the percent growth inhibition induced by siHsp27 treatment of PTEN-deficient PC-3 cells was assessed in cells grown in the presence of varying concentrations of the potent PI3K inhibitor, LY294002. As shown in Figure 7e, LY294002 treatment reduced growth inhibitory effect induced by siHsp27 treatment in a dose-dependent manner. These data demonstrate that growth inhibitory effects induced by $\mathrm{Hsp} 27$ silencing may be influenced by the activation status of the PTEN/PI3K/Akt pathway.

\section{Discussion}

Previous studies have shown that PEA-15 can regulate both the ERK/MAPK pathway and the death receptor-initiated pathway as a result of PEA-15 binding to ERK or FADD, respectively. ${ }^{14}$ Akt-mediated phosphorylation of PEA-15 switches the binding specificity of PEA-15 from ERK to FADD, resulting in enhanced proliferation by releasing ERK and allowing its nuclear translocation, while also inhibiting Fas-induced apoptosis via binding and sequestering FADD. Herein, we show that Hsp27 has a critical role in regulating cell proliferation and Fas-induced cell death. The results presented here are consistent with a model (Figure 8) wherein the association of Hsp27 with Akt leads to stabilization and activation of Akt, which, in turn, phosphorylates PEA-15 at Ser-116, switching the binding specificity of PEA-15 from ERK to FADD. The dissociation of PEA-15 from ERK alleviates the cytoplasmic sequestration of ERK, allowing its translocation into the nucleus where it mediates its mitogenic effects. Upon phosphorylation of PEA-15 at Ser-116, PEA-15 binds to FADD and thereby inhibits Fas-induced apoptosis.

Here, we show that Hsp27 interacts with Akt in prostate cancer cells. These findings are consistent with previous reports by Konishi et al. ${ }^{20}$ and Rane et al. ${ }^{21}$ demonstrating that Hsp27 interacts with and promotes activation of Akt in COS-7 kidney cells and neutrophils. Previous findings by Beguinot and coworkers have shown that Akt associates with and stabilizes PEA-15 and that PEA-15 is a downstream substrate of $A k t,{ }^{12}$ suggesting that binding of $\mathrm{Hsp} 27$ with Akt promotes its stability and activity, and in turn Akt binds, stabilizes and phosphorylates PEA-15 at Ser-116. Consistent with this notion, we find that increased Hsp27 levels correlate with elevated levels of total PEA-15 and phospho-Ser-116PEA-15.

Hsp27 has been shown to be capable of inhibiting extrinsic cell death triggered by engagement of the Fas death receptor. $^{22}$ To date, death-associated protein 6 (Daxx) is the only Hsp27-regulated component of the extrinsic death pathway that has been identified. Hsp27 has been shown to bind to Daxx, preventing the interaction of Daxx with apoptosis signal-regulating kinase 1 (Ask1) and Fas, and inhibiting Daxx-induced apoptosis. ${ }^{23}$ Daxx is a death receptor-binding protein that mediates an alternative, FADD- and caspaseindependent death pathway via Ask1 and c-Jun N-terminal kinase. Although Daxx is an important mediator of Fasinduced apoptosis, the ability of Hsp27 to inhibit Daxx alone cannot fully explain the ability of Hsp27 to inhibit the classical Fas-induced FADD- and caspase-dependent death pathway. ${ }^{22}$ Here, we show that Hsp27 suppresses Fas-induced apoptosis via promoting FADD-PEA-15 interaction, and that the anti-apoptotic activity of Hsp27 correlates with enhanced phosphorylation of PEA-15 at Ser-116. This is consistent with
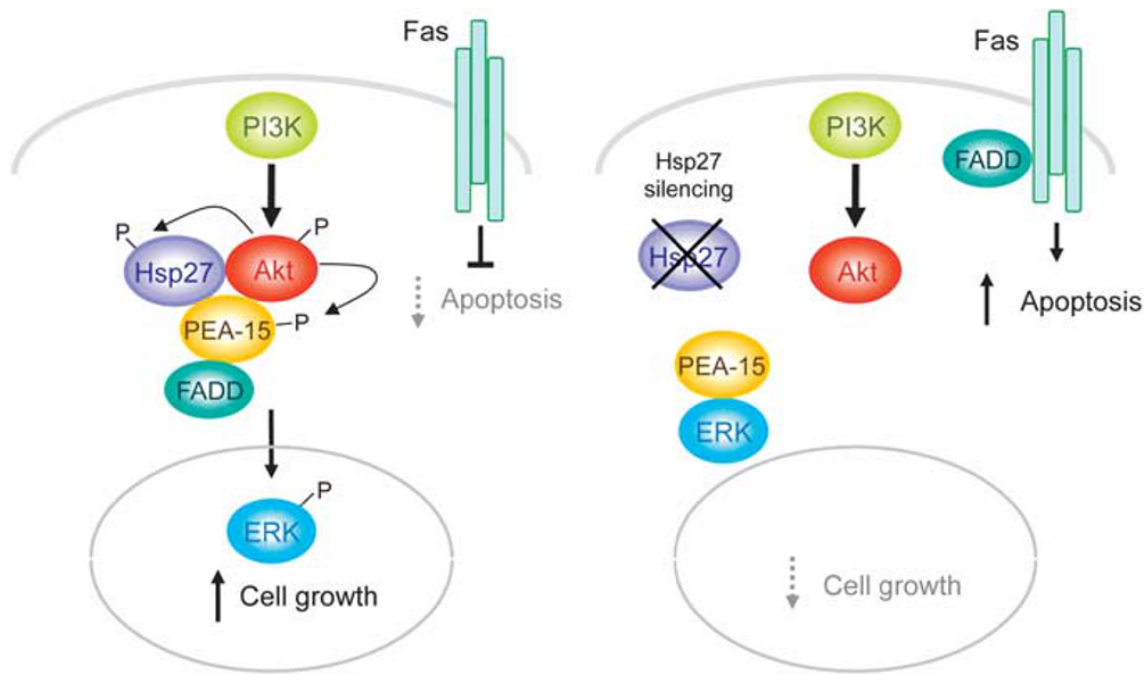

Figure 8 A proposed model for Hsp27/Akt/PEA-15 signaling in the regulation of Fas-mediated apoptosis and cell proliferation in PTEN-deficient cells with (right) and without (left) Hsp27 silencing 
previous findings that PEA-15 regulates FADD in a phosphorylation-dependent manner.

Despite extensive knowledge of the cytoprotective properties of Hsp27, relatively little is known about the role of Hsp27 in regulating cell proliferation. Here, we show that Hsp27 regulates ERK nuclear translocation in a PEA-15-dependent manner. Ginsberg and coworkers have shown that PEA-15 is a key regulator of ERK nuclear translocation through its ability to directly bind to and sequester ERK in the cytoplasm. ${ }^{8,10}$ Another mechanism by which Hsp27 has been proposed to impact the cell cycle is through its ability to regulate the ubiquitination and degradation of p27kip $1 .{ }^{24}$ Consistent with this, we have found that p27kip1 levels are downregulated in Hsp27-overexpressing LNCaP cells. The ability of Hsp27 to regulate Akt activity may also contribute to the overall cytoprotective and proliferative functions of $\mathrm{Hsp} 27^{11}$ by regulating the function of pro-apoptotic proteins, such as BAD and caspase-9, cell cycle regulators, such as p27kip1, and mediators that control apoptosis and/or proliferation, such as elF4E, MDM2, FOXO, GSK3, TSC2 and PRAS40. ${ }^{11,25}$

The concept of oncogenic pathway addiction ${ }^{19}$ implies that reversal of one or a few genes can inhibit cancer cell growth and in some cases translate into improved clinical responses. Our findings that Hsp27 silencing preferentially inhibits growth of PTEN-deficient cancer cells suggest that Hsp27 silencing and its downstream effects on Akt may show more pronounced effects on PTEN-deficient cancer cells because of the acquired dependence or addiction of these cells on the $\mathrm{PI} 3 \mathrm{~K} / \mathrm{Akt}$ pathway for their growth and survival. These findings suggest that patients with tumors harboring abnormalities in PTEN function may clinically respond more favorably to OGX427 , thus identifying a population more likely to benefit from Hsp27 inhibition therapy.

\begin{abstract}
Materials and Methods
Cell lines and materials. LNCaP, PC-3, DU145, 293T, Ku7, RT4, UMUC3 and MDA468 cells were purchased from American Type Culture Collection (Rockville, MD, USA). PNT1b provide by Professor N Maitland (York, UK); LAPC4 provided by Dr. Rob Reiter at UCLA, Los Angeles, CA, USA; and BPH-1 cells were a gift from Dr. Simon Hayward, UCSF, San Francisco, CA, USA. LNCaP (used up to passage 50 in the present study), DU145, LAPC4, BPH-1 and PNT1b cells were routinely maintained in RPMI1640 (Life Technologies, Burlington, ON, Canada). RT4 cells were maintained in McCoy's media (Life Technologies). Other cells were maintained in DMEM (Life Technologies). Media were supplemented with $10 \%$ fetal bovine serum (FBS), and cultures were grown at $37^{\circ} \mathrm{C}$ and $5 \% \mathrm{CO}_{2}$. GSK690693C, a potent small-molecule Akt inhibitor, was kindly provided by Dr. Rakesh Kumar. $\mathrm{CH}-11$, anti-Fas antibody, was purchased from Upstate (Billerica, MA, USA). CHX, Dox and LY294002 were purchased from Sigma-Aldrich (Oakville, ON, USA). Antibodies against Hsp27, phospho-Hsp27 (Ser-82) (StressGen, Victoria, BC, USA), PEA-15 (Santa Cruz Biotechnology, Santa Cruz, CA, USA), phospho-PEA15 (Ser-116; Biosource, Burlington, ON, Canada), Akt, phospho-Akt (Ser-473), phospho-Foxo-1 (Ser-256; Cell Signaling Technology, Danvers, MA, USA), FADD (Upstate), p27kip1, cyclin D1, CDK2 (Santa Cruz Biotechnology) and vinculin (Sigma-Aldrich) were used according to manufacture's instructions.
\end{abstract}

Lentiviral transduction of LNCaP cells. Two vectors, $\mathrm{pHR}^{\prime}$ cytomegalovirus (CMV)-Hsp27 and pHR'-CMV as an empty vector, were used in the present study as previously described. ${ }^{4} \mathrm{pHR}^{\prime}-\mathrm{CMV}$-Hsp27 contains the fulllength CDNA for human Hsp27 subcloned into the BamHI and Xhol sites of the pHR'-CMV-enhanced green fluorescent protein (GFP) lentiviral vector. Lentiviruses were prepared and transduced into LNCaP cells as described. ${ }^{4}$ Expression of GFP and Hsp27 in transduced LNCaP cells was verified by fluorescence microscopy and western blotting, respectively. Lentiviral FLAG-tagged PEA-15 (pLB-U-FPEA-15WT) was derived by PCR and Gateway cloning using the Human PEA-15 cDNA clone (TC108132, OriGene Technologies Inc., Rockville, MD, USA) as template and attB1 and attB2 containing forward 5'-GGGGACAAGTTTGTACAAAAAAGCA GGCTTCACCATGGATTATAAAGATGATGACGATAAAGCTGAGTACGGGACCC TCCTG-3' and reverse $5^{\prime}$-GGGGACCACTTTGTACAAAGCTGGGTCTCAGGCCT TCTTCGGTGG-3' primers, respectively. FLAG-tagged PEA-15 S116D (pLB-UFPEA-15 S116D) was generated by site-directed mutagenesis of Human PEA-15 cDNA clone using the Quick change site-directed mutagenesis kit (Stratagene, La Jolla, CA, USA) according to the manufacturer's suggested protocol with forward 5'- CATTATCCGGCAGCCCGATGAGGAAGAGATCATCAAATTGGC-3' and reverse 5'-GCCAATTTGATGATCTCTTCCTCATCGGGCTGCCGGATAATG-3' primers, respectively, followed by the Invitrogen Gateway cloning technology (Carlsbad, CA, USA) using the PCR primers described above. FLAG-tagged PEA-15 S116G containing seven silent mutations within nucleotides 221-255 were synthesized with flanking $5^{\prime}$-EcoR1-attB1 and $3^{\prime}$-Hindll-attB2 sites and ligated to pUC57 by Genscript Corporation (Piscataway, NJ, USA). These constructs were generated using a modified lentiviral expression plasmid (FUGW), ${ }^{26}$ where the GFP protein was replaced by the Gateway cassette and a blasticidin resistance gene expression module was inserted downstream of the Gateway cassette (pLB-U). All constructs were confirmed by DNA sequence analysis.

siRNA-mediated gene silencing. LNCaP cells were plated at $7 \times 10^{5}$ per $10 \mathrm{~cm}$ dish. After $24 \mathrm{~h}$, cells were transfected with siHsp27 or Scr duplexes as previously described. ${ }^{27}$ Briefly, the RNA duplex was diluted in Opti-MEM I serumfree medium and Oligofectamin (Invitrogen) and incubated at room temperature for $20 \mathrm{~min}$ before addition to cells. The media was changed after $4-6 \mathrm{~h}$. Cells were used $48 \mathrm{~h}$ after transfection. The following siRNAs were used: siAkt (Cell Signaling Technology), siHsp27 (5'-AAGUCUCAUCGGAUUUUGCAGC-3'; Dharmacon, Lafayette, CO, USA) and Scr (5'-CAGCGCUGACAACAGUUUCAU- $\left.3^{\prime}\right)$.

Cell proliferation assay. Cells were seeded at $0.5 \times 10^{5}$ cells per well in six-well dishes. At $24 \mathrm{~h}$ after culture, cell growth was quantified by direct cell counting at 2-day intervals up to 7 days. Each experiment was repeated three times. Alternatively, cell number was quantified using the Invitrogen CyQuant cell proliferation assay as per the manufacturer's protocols.

$\left[{ }^{3} \mathrm{H}\right]$-thymidine incorporation assay. Cells were seeded at $4 \times 10^{4} \mathrm{celll} / \mathrm{ml}$ in 12-well plates in normal growth media for $24 \mathrm{~h}$. At 24 or $48 \mathrm{~h}$ after culture, $10 \mu \mathrm{l}$ of $100 \mu \mathrm{Ci} / \mathrm{ml}\left[{ }^{3} \mathrm{H}\right]$-thymidine was added per well and cells were incubated for $3 \mathrm{~h}$. The cells were detached from the plate with a trypsin-EDTA solution $(0.05 \%$ trypsin and $0.53 \mathrm{mM}$ EDTA; Life Technologies Inc., Gaithersburg, MD, USA). After

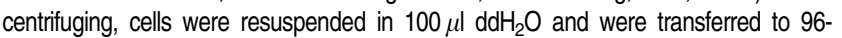
well plates. The collected cells were harvested onto glass-fiber filter mats using a Tomtec Harvester 96 Mach 3 M (Hamden, CT, USA) and counted on a Wallac 1450 Microbeta plate scintillation counter (Turku, Finland). Each experiment was performed six times.

Flow cytometric bromodeoxyuridine incorporation assay. 5-Bromo-2'-deoxyuridine (BrdU) is a synthetic nucleoside analog of thymidine. $\mathrm{BrdU}$ is incorporated into the newly synthesized DNA of replicating cells (during the $S$ phase of the cell cycle), substituting for thymidine during DNA replication. Cells were incubated with $10 \mu \mathrm{M} \mathrm{BrdU}$ for $2 \mathrm{~h}$, and intracellular staining for BrdU incorporation was performed as per the manufacturer's instructions (BrdU Flow kit; BD Biosciences, San Jose, CA, USA). The percentage of cells undergoing DNA replication was determined by flow cytometry.

Immunofluorescence microscopy. Cells were grown on glass coverslips in standard media for $24 \mathrm{~h}$. Cells were fixed with cold $3 \%$ acetone in methanol for $10 \mathrm{~min}$ at $-20^{\circ} \mathrm{C}$ and permeabilized in $0.2 \%$ Triton in phosphate-buffered saline (PBS). Slides were incubated in blocking solution, $5 \%$ bovine serum albumin in PBS for $1 \mathrm{~h}$ and simultaneously treated overnight with primary antibodies, mouse monoclonal Hsp27 and rabbit polyclonal ERK antibodies. Secondary fluorescent antibodies, anti-mouse fluorescein isothiocyanate and anti-rabbit Texas Red Conjugated were added for $1 \mathrm{~h}$ at room temperature with three 5 -min washes $(0.1 \%$ Triton in PBS). Cells were counterstained and mounted with coverslips using Vectashield mounting medium containing $4^{\prime}, 6^{\prime}$-diaminido-2-phenylindole (DAPI; Vector Laboratories, Burlingame, CA, USA). Images were captured using a Zeiss Axioplan II fluorescence microscope (Carl Zeiss Instruments, Oberkochen, Germany) at $\times 100$ magnification, followed by analysis with imaging software 
(Northern Eclipse, Empix Imaging Inc., Mississauga, ON, Canada). To more objectively quantify the nuclear-cytoplasmic distribution of ERK, monochromatic high-resolution fluorescence images were acquired for at least 20 cells from each experimental condition. The representative images were converted to TIFF files and used to determine the ratio of fluorescence in the nucleus relative to the cytoplasm (FN/C), using the Northern Eclipse (Empix Imaging Inc.) and Adobe Photoshop CS software (Adobe Systems, San Jose, CA, USA). The DAPI signal was then used to identify cell nuclei and the mean pixel density was assessed for manually cropped areas of uniform intensity in both nucleus and cytoplasm, and ratios were determined.

Generation of PEA-15-targeted shRNA. shRNA targeting PEA-15 nucleotides 221-250 (Genbank accession number NM_0037568) was designed, and corresponding DNA oligonucleotides were synthesized and cloned into the BseR1 and BamH1 sites of pSHAG-1(Invitrogen) to generate a PEA-15 shRNA expression cassette driven by the U6 polymerase III promoter. The PEA-15 shRNA expression cassette was then subcloned via Gateway cloning technology (Invitrogen) into the BLOCK-iT lentiviral RNAi expression destination vector pLenti6, to generate the final expression plasmid pLenti6 PEA-15 221 (PEA-15 221). PEA-15 shRNA-mediated silencing of endogenous PEA-15 expression was confirmed by quantitative real-time PCR and immunoblotting

Immunoprecipitation. Cell lysates were incubated with $5 \mu \mathrm{g}$ anti-Akt, antiPEA-15 or anti-lgG antibodies. After $12 \mathrm{~h}$ of incubation, $50 \mu \mathrm{l}$ of protein A/G beads (Amersham Pharmacia Biosciences, Piscataway, NJ, USA) was added into the reaction tubes and incubated for $2 \mathrm{~h}$. The beads were washed three times using $1 \times$ PBS and resolved in $2 \times$ loading buffer (MBI, Fermentas Inc., Burlington, ON, Canada). The indicated antibody was used, and bands were detected as described above.

In vitro Akt kinase assay. Akt kinase activity was measured using an Akt kinase assay kit (Cell Signaling Technology) according to the manufacture's instructions. Briefly, Akt was immunoprecipitated from cells by incubating cell lysates (500 $\mu \mathrm{g}$ of protein) at $4{ }^{\circ} \mathrm{C}$ rotating overnight with protein G-agarose beads bearing anti-Akt antibody. After washing, $1 \mu \mathrm{g}$ of recombinant GSK3 $\alpha / \beta$ was added to the beads in the presence of magnesium/ATP mixture and incubated for $30 \mathrm{~min}$ at $30^{\circ} \mathrm{C}$. SDS sample buffer was then added, samples were boiled for $5 \mathrm{~min}$ and electrophoresed on $10 \%$ SDS-PAGE. The membranes were probed with antiGSK3 $\alpha / \beta$ (Ser-21/9) and anti-Akt antibodies. Equal amounts of input cell protein were verified by immunoblotting for vinculin.

Protein stability. Cells were cultured in normal growth media for $18-24 \mathrm{~h}$ before changing the media to $5 \%$ FBS containing RPMI $+10 \mu \mathrm{g} / \mathrm{ml} \mathrm{CHX.} \mathrm{Cell}$ lysates were prepared after culturing cells for the indicated lengths of time, and Akt, Hsp27 and GAPDH protein levels were assessed by immunoblotting.

Elk-1 transcription reporter assay. Cells were seeded into 12-well plates at $10^{5}$ cells per well and were transiently cotransfected in Opti-MEM I medium with lipofectin (Invitrogen) with $0.5 \mu \mathrm{g}$. GAL-E1b-luciferase reporter gene and varying doses of pCMV-GAL4-Elk-1 kindly provided by Professor Richard A Maure (OR, USA). Samples were normalized by cotransfection with pCMV-Renilla luciferase. Sixteen hours after transfection, media were replaced with RPMI 1640 plus $10 \% \mathrm{FBS}$, and cell lysates were prepared $48 \mathrm{~h}$ later. Luciferase activities in cell lysates were quantified using the Dual Luciferase Assay system (Promega, Madison, WI, USA) with the aid of a MicroLumat Plus luminometer (EG\&G Berthold, Bad Wildbad, Germany). Reporter assays were expressed in arbitrary light units and performed three times.

Fas-induced apoptosis assay. Cells were treated by either $2.5 \mu \mathrm{g} / \mathrm{ml} \mathrm{CHX}$ alone, $1.0 \mu \mathrm{g} / \mathrm{ml}$ anti-Fas antibody (CH-11) alone or in combination in $5 \%$ charcoalstripped serum media. Twenty hours later, cells were harvested and the percentage of cells with sub-G0/G1 DNA content was determined by flow cytometry as previously described. ${ }^{3}$ In other experiments, cells were transfected with $20 \mathrm{nM} \mathrm{Scr}$ or siHsp27 and treated with $1.0 \mu \mathrm{g} / \mathrm{ml} \mathrm{CH}-11$ in low-serum (0.5\% FBS) media $48 \mathrm{~h}$ after transfection. The proportion of cells containing sub-G0/G1 DNA content after $20 \mathrm{~h}$ of $\mathrm{CH}-11$ treatment was determined as a measure of apoptosis as described above. Each experiment was performed three times.

Statistical analysis. All results were expressed as the mean \pm S.D. Statistical analysis was performed with a one-way ANOVA, followed by Fisher's protected least significant difference test (StatView 512, Brain Power Inc., Calabasas, CA USA). ${ }^{\star} P<0.05$ was considered significant.

\section{Conflict of Interest}

This work was supported by grants from the National Cancer Institute of Canada, NIH Pacific Northwest Prostate SPORE and Canadian Institutes of Health Research.

Acknowledgements. This work was funded by the Cancer Research Society (CJO), Canadian Institutes of Health Research (CJO), NIH Pacific Northwest Prostate Cancer SPORE (MEG) and a NCIC Terry Fox New Frontiers Program Project Grant (MEG)

1. Garrido $C$, Brunet M, Didelot $C$, Zermati $Y$, Schmitt $E$, Kroemer $G$. Heat shock proteins 27 and 70: anti-apoptotic proteins with tumorigenic properties. Cell Cycle 2006; 5: 2592-2601.

2. Ciocca DR, Calderwood SK. Heat shock proteins in cancer: diagnostic, prognostic, predictive, and treatment implications. Cell Stress Chaperones 2005; 10: 86-103.

3. Rocchi P, So A, Kojima S, Signaevsky M, Beraldi E, Fazli L et al. Heat shock protein 27 increases after androgen ablation and plays a cytoprotective role in hormone-refractory prostate cancer. Cancer Res 2004; 64: 6595-6602.

4. Rocchi P, Beraldi E, Ettinger S, Fazli L, Vessella RL, Nelson C et al. Increased Hsp27 after androgen ablation facilitates androgen-independent progression in prostate cancer via signal transducers and activators of transcription 3-mediated suppression of apoptosis. Cancer Res 2005; 65: 11083-11093.

5. Kamada M, So A, Muramaki M, Rocchi P, Beraldi E, Gleave M. Hsp27 knockdown using nucleotide-based therapies inhibit tumor growth and enhance chemotherapy in human bladder cancer cells. Mol Cancer Ther 2007; 6: 299-308.

6. Garrido C, Fromentin A, Bonnotte B, Favre N, Moutet M, Arrigo AP et al. Heat shock protein 27 enhances the tumorigenicity of immunogenic rat colon carcinoma cell clones. Cancer Res 1998; 58: 5495-5499.

7. Rocchi $P$, Jugpal $P$, So A, Sinneman S, Ettinger S, Fazli $L$ et al. Small interference RNA targeting heat-shock protein 27 inhibits the growth of prostatic cell lines and induces apoptosis via caspase-3 activation in vitro. BJU Int 2006; 98: 1082-1089.

8. Formstecher E, Ramos JW, Fauquet M, Calderwood DA, Hsieh JC, Canton B et al. PEA-15 mediates cytoplasmic sequestration of ERK MAP kinase. Dev Cell 2001; 1: 239-250.

9. Gaumont-Leclerc MF, Mukhopadhyay UK, Goumard S, Ferbeyre G. PEA-15 is inhibited by adenovirus E1A and plays a role in ERK nuclear export and Ras-induced senescence. J Biol Chem 2004; 279: 46802-46809.

10. Hill JM, Vaidyanathan H, Ramos JW, Ginsberg MH, Werner MH. Recognition of ERK MAP kinase by PEA-15 reveals a common docking site within the death domain and death effector domain. EMBO J 2002; 21: 6494-6504.

11. Manning BD, Cantley LC. AKT/PKB signaling: navigating downstream. Cell 2007; 129: 1261-1274.

12. Trencia A, Perfetti A, Cassese A, Vigliotta G, Miele C, Oriente F et al. Protein kinase B/Akt binds and phosphorylates PED/PEA-15, stabilizing its antiapoptotic action. Mol Cell Biol 2003; 23: 4511-4521.

13. Rhodes N, Heerding DA, Duckett DR, Eberwein DJ, Knick VB, Lansing TJ et al. Characterization of an Akt kinase inhibitor with potent pharmacodynamic and antitumor activity. Cancer Res 2008; 68: 2366-2374.

14. Renganathan $H$, Vaidyanathan $H$, Knapinska $A$, Ramos JW. Phosphorylation of PEA-15 switches its binding specificity from ERK/MAPK to FADD. Biochem J 2005; 390: 729-735.

15. Rokhlin OW, Bishop GA, Hostager BS, Waldschmidt TJ, Sidorenko SP, Pavloff N et al. Fas-mediated apoptosis in human prostatic carcinoma cell lines. Cancer Res 1997; 57: 1758-1768.

16. Sebolt-Leopold JS, Herrera R. Targeting the mitogen-activated protein kinase cascade to treat cancer. Nat Rev Cancer 2004; 4: 937-947.

17. Parsons R. Human cancer, PTEN and the PI-3 kinase pathway. Semin Cell Dev Biol 2004; 15: $171-176$.

18. Weinstein IB, Joe AK. Mechanisms of disease: oncogene addiction-a rationale for molecular targeting in cancer therapy. Nat Clin Pract Oncol 2006; 3: 448-457.

19. Weinstein IB, Joe A. Oncogene addiction. Cancer Res 2008; 68: 3077-3080; discussion 3080 .

20. Konishi $\mathrm{H}$, Matsuzaki $\mathrm{H}$, Tanaka $\mathrm{M}$, Takemura $\mathrm{Y}$, Kuroda $\mathrm{S}$, Ono $\mathrm{Y}$ et al. Activation of protein kinase $B$ (Akt/RAC-protein kinase) by cellular stress and its association with heat shock protein Hsp27. FEBS Lett 1997; 410: 493-498.

21. Rane MJ, Pan Y, Singh S, Powell DW, Wu R, Cummins T et al. Heat shock protein 27 controls apoptosis by regulating Akt activation. J Biol Chem 2003; 278: 27828-27835.

22. Mehlen $\mathrm{P}$, Schulze-Osthoff $K$, Arrigo AP. Small stress proteins as novel regulators of apoptosis. Heat shock protein 27 blocks Fas/APO-1- and staurosporine-induced cell death. J Biol Chem 1996; 271: 16510-16514.

23. Charette SJ, Lavoie JN, Lambert H, Landry J. Inhibition of Daxx-mediated apoptosis by heat shock protein 27. Mol Cell Biol 2000; 20: 7602-7612. 
24. Parcellier A, Brunet M, Schmitt E, Col E, Didelot C, Hammann A et al. HSP27 favors ubiquitination and proteasomal degradation of p27Kip1 and helps S-phase re-entry in stressed cells. FASEB J 2006; 20: 1179-1181.

25. Andrieu C, Taieb D, Baylot V, Ettinger S, Soubeyran P, De-Thonel A et al. Heat shock protein 27 confers resistance to androgen ablation and chemotherapy in prostate cancer cells through elF4E. Oncogene 2010; 29: 1883-1896.
26. Lois C, Hong EJ, Pease S, Brown EJ, Baltimore D. Germline transmission and tissue-specific expression of transgenes delivered by lentiviral vectors. Science 2002; 295: 868-872.

27. Trougakos IP, So A, Jansen B, Gleave ME, Gonos ES. Silencing expression of the clusterin/apolipoprotein j gene in human cancer cells using small interfering RNA induces spontaneous apoptosis, reduced growth ability, and cell sensitization to genotoxic and oxidative stress. Cancer Res 2004; 64: 1834-1842. 